\title{
Efeito de Sistemas de Alimentação no Ganho de Peso e Desempenho Reprodutivo de Novilhas Nelore Acasaladas aos 17/18 Meses
}

\author{
Cláudio Eduard Neves Semmelmann², José Fernando Piva Lobato ${ }^{3}$, Marta Gomes da Rocha ${ }^{4}$
}

RESUMO - Foram avaliados, por intermédio do modelo estatístico linear (GLM/SAS), o crescimento e o desempenho reprodutivo de 480 bezerras Nelore dos 7,5 meses até o acasalamento aos 17/18 meses de idade em quatro sistemas alimentares no período seco (05 a 10/96): Brachiaria brizantha cv. Marandu (B), Brachiaria brizantha cv. Marandu + Anipro (BA), milheto (Pennisetum americanum cv. (L.) Leeke) (M) e Brachiaria brizantha cv. Marandu + Sal Ouro (BS). A partir de 10/96, em lote único, pastejaram em Panicum maximum cv. Mombaça, $1 \mathrm{UA} / \mathrm{ha}$, até o fim do acasalamento (03/97 a 05/97). Foram avaliados o peso vivo (PV), condição corporal (CC), ganho médio diário (GMD), idade ao acasalamento (IAC), taxa de prenhez (TP) e idade da mãe (IM), além de escores de conformação (Cs), precocidade (Ps) e musculosidade (Ms). Os sistemas BA e M apresentaram diferença significativa com maior PV, GMD e CC após o período da estação seca em relação aos sistemas B e BS. No início do acasalamento, o PV dos sistemas B, BA e M diferiram do grupo BS. Ao final do acasalamento, não foram detectadas diferenças em GMD, CC, IAC e IM entre os sistemas. Os tratamentos B, BA, M e BS utilizados durante a estação seca não afetaram, respectivamente, a TP média de 22,2;24,2; 20,7; e 13,8\%. Houve diferença significativa entre novilhas prenhas e falhadas em relação a PV, CC, IAC, GMD, Cs, Ps e Ms.

Palavras-chave: acasalamento aos 17/18 meses, Nelore, sistemas de recria

\section{Effect of Feeding Systems on Weight Gain and Reproductive Performance of Nellore Heifers Bred at the Age of 17/18 Months}

\begin{abstract}
Using a linear statistical model (GLM/SAS) the reproductive performance and growth of 480 Nellore heifers were evaluated from the age of 7.5 to 17/18 months under four feeding systems during the dry period (96/05 to 96/10): Brachiaria brizantha cv. Marandu pasture (B); Brachiaria brizantha cv. Marandu pasture + Anipro (BA); Pennisetum americanum cv. (L.) Leeke (Pearlmillet) (M) and Brachiaria brizantha cv. Marandu pasture + Sal Ouro (BS). At the end of supplementation all heifers were moved to a paddock of Panicum maximum cv. Mombaça pasture, 1 AU/ha, from 96/10 until the end of the mating season (97/03 to 97/05). Parameters like liveweight (LW), body condition score (BCS), liveweight daily gain (LDG), age at mating (AM), pregnancy rate (PR) and age of the dam (AD) were determined. Besides these variables pregnant and non-pregnant heifers were also evaluated for scores of conformation (Cs), precocity (Ps) and muscularity (Ms). The systems BA and M registered significantly differences with major LW, LDG and BCS values after the dry season compared to systems B and BS. At the beginning of the mating season LW values from B, BA and M were different of BS system. At the end of the mating season no differences could be detected among the four systems regarding LDG, BCS, $\mathrm{AM}$, and AD. No significant effect on the PR averages of the four feeding systems B, BA, M e BS was registered $(22,2 \%, 24,2 \%, 20,7 \%$ e 13,8\%). Significant differences $(\mathrm{P}<0.01)$ between pregnant and non-pregnant Nellore heifers could be detected for LW, BCS, AM, LDG, Cs, Ps, and Ms.
\end{abstract}

Key Words: mating at $17 / 18$ months, Nellore, heifer feeding systems

\section{Introdução}

O desenvolvimento de fêmeas de reposição é uma fase crítica em sistemas de produção de pecuária de corte. A idade média ao primeiro parto na pecuária de corte no Brasil está acima de 40 meses da idade, conforme relato de PEREIRA (2000), sendo a alimentação inadequada a principal causa desta situação. Significativa proporção do rebanho bovino brasileiro é constituída de fêmeas jovens de um a três anos de idade. Segundo FRIES (1998), para cada 1000 vacas em produção, existem cerca de 800 em recria, ocupando pelo menos a metade da área ocupada por aquelas. Conseqüentemente, a quantidade de área e custos de mantença alocados para a recria de fêmeas são consideráveis. As exigências

\footnotetext{
${ }^{1}$ Parte da dissertação de mestrado do primeiro autor.

2 Médico Veterinário (UFRGS) e mestre em Zootecnia (UFRGS). E.mail: semmdvm@terra.com.br

3 Professor Adjunto IV, Departamento de Zootecnia, Fac. de Agronomia (UFRGS), Pesq. CNPq - 1A, Caixa Postal, 776, $90001-970$ - Porto Alegre, RS.

E.mail: lobato@orion.ufrgs.br

4 Professora do Departamento de Zootecnia (UFSM) - 97119-900 - Santa Maria, RS. E.mail: tata@pro.via-rs.com.br
} 
nutricionais anuais de fêmeas em recria e adultas constituem dois terços do total de alimentos de um sistema de produção de bovinos de corte nos Estados Unidos (FERREL e JENKINS, 1988).

O objetivo principal de um sistema de recria de reposição é desenvolver novilhas que tenham alcançada a puberdade e ciclem regularmente antes do início da primeira estação de acasalamento. As falhas no manejo e planejamento da alimentação, durante os meses de menor oferta de forragem (estação seca ou hibernal), podem levar a aumento da idade a puberdade, baixa taxa de prenhez, maior número de partos distócicos, maior morbidade e mortalidade de bezerros, baixos pesos à desmama, redução da vida produtiva da fêmea e baixos índices de reconcepção quando primíparas. Os fatores ambientais, o peso vivo, os ganhos de peso pré e pósdesmama, a condição corporal e idade à puberdade são pontos chaves na redução da idade ao primeiro acasalamento (PATTERSON et al., 1992; LOBATO, 1998). A utilização do acasalamento aos 18 meses pode ser um passo intermediário entre o sistema de primeiro serviço aos 13-15 meses de idade (sistema um ano) e aos 25-26 meses (sistema dois anos). A identificação de fêmeas da raça Nelore que concebam em idades mais jovens é uma das prioridades de alguns programas de seleção e melhoramento da raça Nelore que visam a redução da idade ao primeiro acasalamento. O objetivo básico no desenvolvimento de fêmeas de reposição é prover a quantidade de ganho adequado ao menor custo possível, levando-se em conta o peso alvo para o acasalamento, idade, biotipo e características auxiliares na identificação de fêmeas mais precoces à puberdade, sistemas de alimentação e/ou suplementação nos períodos de escassez. A utilização da suplementação e a combinação de diferentes espécies forrageiras de maior potencial de ganho de peso em distintas épocas podem proporcionar melhores ganhos de peso para sistemas de produção de carne no Brasil Central. Durante o período seco, as características químicas das forrageiras (baixo conteúdo de nitrôgenio, baixa digestibilidade e alta fibra) limitam o consumo. A inclusão de pastagens de milheto (Pennisetum americanum) e/ou a utilização de suplementos (líquidos ou secos) à base de nitrogênio não protéico (NNP), dentro de um sistema de produção, surgem como alternativas para garantir o desempenho alvo em períodos críticos.

O objetivo deste trabalho foi avaliar quatro sistemas de alimentação de novilhas Nelore em termos de desempenho produtivo e reprodutivo entre a idade de
7,5 meses, até o final do primeiro acasalamento aos 17/18 meses. Também foram analisadas diferenças de desempenho nestes parâmetros, entre novilhas Nelore prenhas e falhadas.

\section{Material e Métodos}

O experimento foi conduzido na Agropecuária Jacarezinho Ltda, no município de Valparaíso, São Paulo. Foram avaliadas 480 bezerras da raça Nelore, da desmama (março/1996) ao término do período de acasalamento aos 17/18 meses de idade (07 de maio de 1997). As bezerras fazem parte de um programa de avaliação genética da raça Nelore iniciado em 1984. Em maio/96, após uma pesagem inicial, as bezerras foram distribuídas aleatoriamente em quatro tratamentos formando lotes homogêneos em relação ao peso vivo. Os quatro sistemas alimentares conduzidos de junho a outubro de 1996 (período seco) foram:

Testemunha: 90 bezerras em pastagem de braquiária (Brachiaria brizantha cv. Marandu) com carga de 1,36 bezerras $/$ ha $=0,55 \mathrm{UA} / \mathrm{ha}=248 \mathrm{~kg} \mathrm{de} \mathrm{PV} / \mathrm{ha}$.

Tratamento BA: 168 bezerras em pastagem de braquiária suplementadas com suplemento líquido (ANIPRO $\left.{ }^{\circledR}\right)$, com carga de 2,21 bezerras $/$ ha $=0,84$ $\mathrm{UA} / \mathrm{ha}=380 \mathrm{~kg}$ de PV/ha. A composição média do suplemento nas suas porções doce e azeda foi, respectivamente, 50,22 e 42,02\% de matéria seca, 15 e $32 \%$ de PB, 38 e $20 \%$ de NDT, 4 e $20 \mathrm{~g} / \mathrm{kg}$ de fósforo, $12 \mathrm{~g} / \mathrm{kg}$ de enxofre, $24 \mathrm{e} 51 \mathrm{~g} / \mathrm{kg}$ de nitrogênio, $8,15 \mathrm{~g} / \mathrm{kg}$ de sódio, $140 \mathrm{mg} / \mathrm{kg}$ de cobre, $500 \mathrm{mg} / \mathrm{kg}$ de zinco, $4,5 \mathrm{mg} / \mathrm{kg}$ de selênio, $4,4 \mathrm{mg} / \mathrm{kg}$ de cobalto, $5,9 \mathrm{mg} / \mathrm{kg}$ de iodo, 25.000 UI/kg de vitamina A, $2700 \mathrm{UI} / \mathrm{kg}$ de vitamina D e $400 \mathrm{UI} / \mathrm{kg}$ de vitamina E. O suplemento fornecido à vontade em dois tanques com a capacidade de $500 \mathrm{~L}$ tinha reposição duas vezes por semana. A quantidade das porções doce e azeda foi de (9:1), com o objetivo de alcançar um consumo médio de $500 \mathrm{~g} / \mathrm{dia} / \mathrm{animal}$.

Tratamento M: 120 bezerras em pastagem de milheto (Pennisetum americanum) em pastejo contínuo de 01/06 a 30/07/96 no potreiro $4 \mathrm{C}_{1}$, de $10 / 08 / 96$ a $14 / 10 / 96$ no potreiro $4 C_{2}$, com carga de 1,62 bezerras $/ \mathrm{ha}=0,65 \mathrm{UA} / \mathrm{ha}=292 \mathrm{~kg} \mathrm{de} \mathrm{PV} / \mathrm{ha}$.

Tratamento $B S: 101$ bezerras em pastagem de braquiária com livre acesso a sal proteinado (Ouro Proteico ${ }^{\circledR} 13 \%$ umidade (máximo), $44 \%$ de PB, $42 \%$ de NDT, $5,4 \%$ de nitrogênio não protéico, $44 \mathrm{~g} \mathrm{de}$ fósforo e 1500 quilocalorias de energia), com carga de 1,2 bezerras $/ \mathrm{ha}=0,5 \mathrm{UA} / \mathrm{ha}=232 \mathrm{~kg} \mathrm{de} \mathrm{PV} / \mathrm{ha}$.

Ao término dos tratamentos experimentais, em 
Rev. bras. zootec.

14/10/1996, as novilhas formaram um lote único em pastagem de Panicum maximum cv. Mombaça (1 UA/ha), em pastejo contínuo, até o fim do acasalamento.

As pesagens, com jejum prévio de 12 horas, foram realizadas, em média, a cada 60 dias a partir 06/1996. Em cada pesagem, as bezerras foram submetidas a avaliações de escores de condição corporal (CC), conforme a classificação de LOWMAN et al. (1976), em que condição 1 = magra; condição $5=$ gorda. Avaliações individuais dos escores visuais de conformação, precocidade e musculosidade (CPM) (escala de 1 a 5; SEVERO, 1994) foram feitas aos 205 (desmama) e 550 dias (sobreano) por três avaliadores.

O período de acasalamento foi de 64 dias (03/03 07/05/97), com $4 \%$ de touros Nelore experientes de 24 meses de idade. O diagnóstico de gestação por palpação retal foi realizado em 20/07/1997.

As estimativas da oferta de pastagem foram feitas pelo método adaptado por GARDNER (1986). Usando o método não destrutivo deste autor, foram feitas avaliações por meio de visualizações e pesagem (dupla amostragem).

A análise estatística foi realizada pelo procedimento GLM (General Linear Model) do pacote computacional SAS (Statistical Analysis System, 1988), versão 6.08. O efeito do tratamento na média de todas as determinações de peso vivo (PV), condição corporal (CC) e GMD das bezerras por fase do experimento, períodos seco (S), das águas (A) e de acasalamento (R) foi examinado por um modelo de parcelas divididas, que considera um erro para tratamentos e outro para os períodos, suas interações e covariáveis. As análises foram efetuadas de acordo com o seguinte modelo matemático:

$$
\begin{gathered}
\mathrm{Y}_{\mathrm{ijklm}}=\mu+\mathrm{T}_{\mathrm{i}}+\mathrm{V}_{\mathrm{j}}+\mathrm{T}_{\mathrm{i}} * \mathrm{~N}_{\mathrm{j}(\mathrm{i})}+\mathrm{P}_{\mathrm{k}}+\mathrm{T}^{*} \mathrm{P}_{(\mathrm{ik})}+\mathrm{p}_{\mathrm{il}} \\
+\mathrm{cc}_{\mathrm{im}}+\mathrm{e}_{\mathrm{ijkl}}
\end{gathered}
$$

em que $Y_{\mathrm{ijklm}}=$ variáveis dependentes; $\mu$ = média de todas as observações; $\mathrm{T}_{\mathrm{i}}=$ efeito do i-ésimo sistema alimentar (tratamento) $\mathrm{V}_{\mathrm{j}}=$ efeito da categoria da mãe da bezerra de índice $\mathrm{j}$, sendo $\mathrm{j}=1$ (primeira cria = nascida no ano de 1992), 2 (segunda cria = nascida no ano de 1991) e 3 (adulta = nascida de 1990 a 1978); $\mathrm{T}_{\mathrm{i}} * \mathrm{~N}_{\mathrm{j}(\mathrm{i})}=$ interação entre i-ésimo tratamento e $\mathrm{j}$-ésima novilha (erro da parcela principal); $\mathrm{P}_{\mathrm{k}}=$ efeito do k-ésimo período; $\mathrm{T}^{*} \mathrm{P}_{(\mathrm{ik})}=$ efeito da interação entre o i-ésimo tratamento e o k-ésimo período; $\mathrm{p}_{\mathrm{il}}=$ covariável peso inicial (PJ) dos sistemas alimentares de ordem $1 ; \mathrm{cc}_{\mathrm{im}}=$ covariável condição corporal no início (CCJ) dos sistemas alimentares de ordem $\mathrm{m} ; \mathrm{e}_{\mathrm{ijklm}}=$ erro aleatório associado a cada observação (erro b).
Todos os efeitos foram considerados fixos. Após análise preliminar, quando as interações não foram significativas $(\mathrm{P}>5 \%)$, estas foram incluídas no erro.

Para as análises por período, as determinações do PV, GMD e CC foram estudadas por intermédio do seguinte modelo matemático:

$$
\mathrm{Y}_{\mathrm{ijk}}=\mu+\mathrm{T}_{\mathrm{i}}+\mathrm{V}(\mathrm{T})_{\mathrm{ij}}+\mathrm{e}_{\mathrm{ijk}}
$$

em que: $\mu=$ média geral comum a todas as observações; $\mathrm{T}_{\mathrm{i}}=$ efeito do i-ésimo tratamento realizado na j-ésima novilha pertencente à k-ésima medida; $\mathrm{V}(\mathrm{T})_{\mathrm{ij}}=\mathrm{a}$ categoria da mãe da j-ésima novilha dentro do sistema alimentar $\mathrm{i} ; \mathrm{e}_{\mathrm{ijk}}=$ erro experimental associado à k-ésima medida realizada no i-ésimo tratamento.

Os dados de prenhez foram analisados pelo teste do qui-quadrado $\left(\chi^{2}\right)$ (GOMES, 1985).

\section{Resultados e Discussão}

Ao início do trabalho, os pesos vivos médios (PJ) foram de 180,6, 180,8, 180,3 e 181,1 kg; a condição corporal (CCJ), de 3,85, 3,85, 3,87 e 3,84; e as idades médias (IDI), de 228, 226, 227 e 227 dias para os tratamentos $\mathrm{B}, \mathrm{BA}, \mathrm{M} \mathrm{e} \mathrm{BS}(\mathrm{P}>0,05)$, respectivamente.

A evolução do GMD entre 31/05/96 e 14/10/96, do peso vivo em outubro e da condição corporal em outubro (CC) no período seco pós-desmama é apresentada na Tabela 1. Os tratamentos alimentares no período seco determinaram diferenças significativas

Tabela 1 - Ganho médio de peso (GMD), médias de peso vivo (PV) e condição corporal (CC) de bezerras Nelore nos quatro sistemas de alimentação, durante a seca pós-desmama (05/96-14/10/96)

Table 1 - Live weight daily gain (LDG), average live weight (LW) and body condition score (BCS) of the Nellore heifers

\begin{tabular}{|c|c|c|c|c|c|}
\hline \multirow[t]{3}{*}{$\begin{array}{l}\text { Parâmetros } \\
\text { Parameters }\end{array}$} & \multicolumn{5}{|c|}{$\begin{array}{l}\text { Tratamentos } \\
\text { Treatments }\end{array}$} \\
\hline & $\mathrm{B}$ & BA & $\mathrm{M}$ & BS & Média \\
\hline & $B$ & $B A$ & $M$ & $B S$ & Mean \\
\hline GMD(kg/dia) & $0,204^{b}$ & $0,249^{\mathrm{a}}$ & $0,282^{a}$ & $0,183^{b}$ & 0,229 \\
\hline $\begin{array}{l}L D G(\mathrm{~kg} / \text { day }) \\
\mathrm{PV}(\mathrm{kg}) 14 / 10 / 96 \\
\text { Liveweight (kg) }\end{array}$ & $208,3^{\mathrm{b}}$ & $214,7^{\mathrm{a}}$ & $218,3^{\mathrm{a}}$ & $205,3^{b}$ & 211,6 \\
\hline $\begin{array}{l}\mathrm{CC}(1-5) 14 / 10 / 96 \\
\text { Body condition sc }\end{array}$ & $3,51^{\mathrm{b}}$ & $3,59^{\mathrm{a}}$ & $3,64^{\mathrm{a}}$ & $3,46^{\mathrm{b}}$ & 3,55 \\
\hline
\end{tabular}
in four feeding systems during the dry season and postweaning period (05/96-10/14/96)

a, b Valores seguidos por letras distintas na mesma linha diferem estatisticamente $(P<0,01)$.

a Valores seguidos por mesma letra na mesma linha não diferem estatisticamente $(P>0,05)$.

$a, b$ Values followed by different letters in the same line statistically differ $(P<.01)$.

a Values followed by the same letter, in the same line, do not statistically differ $(P>.05)$. 
( $\mathrm{P}<0,01)$, quando comparados os sistemas BA e M com os sistemas B e BS. O GMD no tratamento M foi 1,$38 ; 1,13$ e 1,54 vezes superior em relação ao $\mathrm{B}, \mathrm{BA}$ e BS, respectivamente, neste período. O estádio de desenvolvimento e a qualidade do milheto permitiram maior facilidade de apreensão e oportunidade de seleção (MORAES, 1984) do que as pastagens de Brachiária com folhas maduras, fibrosas e mortas. Os teores médios de PB na matéria seca das pastagens de Brachiária (B, BA e BS) obtidas pelo método simulando pastejo - considerando planta inteira, por um lado, e somente matéria verde, por outro, e milheto (planta inteira) (M) foram, respectivamente, de 3,08; 5,58 e $14,08 \%$ em agosto e 5,43; 6,98 e 16,20\% em outubro. Os resíduos médios de matéria seca das pastagens de Brachiária (B, BA e BS) e milheto (M) foram de 1510 e $1640 \mathrm{~kg} / \mathrm{MS}$ em agosto e 4289 e 1700 $\mathrm{kg} / \mathrm{MS}$ em outubro. No sistema BA com um consumo médio de $0,380 \mathrm{~kg} /$ dia do suplemento, pode-se observar melhora do ganho médio diário em comparação ao grupo B. A inclusão de suplementos líquidos à base de melaço com uréia tem sido recomendada para a manutenção e redução de perdas de peso em animais adultos pela inferência de resultados obtidos em experimentos que avaliam o consumo de forragens de baixa qualidade em galpões de estudos metabólicos. O baixo consumo do suplemento no sistema BS não conseguiu prover nutrientes suficientes para estimular o consumo da pastagem de brachiária durante $o$ período seco.

Os GMD do lote M durante o período seco foi de $0,282 \mathrm{~kg}$, o qual é inferior duas a três vezes aos valores observados por DUARTE (1980) e MORAES et al. (1995) em pastagens intensamente adubadas no período de primavera/verão no Estado do Rio Grande do Sul. A duração dos dias, temperaturas médias diurnas e noturnas e, principalmente, ocorrência de chuvas e resistência à seca e a adubação são pontos chave que podem ter afetado o desempenho do tratamento M (MARASCHIN, 1981).

O sistema M teve efeito semelhante a um suplemento protéico/energético, apesar das plantas apresentarem o estádio pré-florecimento e florecimento, quando foram pastejadas. A influência do regime de precipitações na área, a implantação da pastagem de milheto dentro de sistemas de reforma de pastagens e rotação via sistema Barreirão e/ou lavoura de soja, além do monitoramento de uma oferta e pressão de pastejo mais moderada, têm o efeito de produzir os ganhos alvos de 0,350-0,450 kg/dia para o acasalamento aos 17/18 meses de idade. ZIMMER
(1987) relata o desempenho de bezerros Nelore com GMD de 0,179 e 0,238 kg em pastagem de Brachiaria brizantha cv. Marandu no período seco (140 dias), com duas lotações de 1,4 e 1,8 UA/ha. Em pastagem de Brachiaria brizantha cv. Marandu, LEITE e EUCLIDES (1994) e EUCLIDES e EUCLIDES FILHO (1997) obtiveram durante o período seco GMD de 0,158 e $0,167 \mathrm{~kg}$, respectivamente. O GMD de B e BS foi superior ao obtido por THIAGO et al. (1997) de $0,148 \mathrm{~kg}$, em Ponta Porã, com bezerros Nelore pósdesmame durante 59 dias (17/05 a 15/07/93).

O consumo médio acumulado do suplemento nos sistemas BA e BS foram, respectivamente, de 380 e $36 \mathrm{~g}$ por dia por cabeça no período de 136 dias. As exigências nutricionais de fêmeas com $180 \mathrm{~kg}$ de peso vivo com um ganho de $0,225 \mathrm{~kg} / \mathrm{dia}$, conforme o NATIONAL RESEARCH COUNCIL - NRC (1984) em relação ao consumo de matéria seca são de 4,1 kg, PB 8,9\% e NDT 56\%. Estas exigências nutricionais indicam que os GMD realizados pelas fêmeas Nelore durante o período seco foram obtidos por melhor oportunidade de seleção da forragem, que apresenta maiores valores de PB e NDT em relação aos valores médios coletados nas avaliações de agosto e outubro/96.

A CC referente ao período junho a agosto/96 foi de 3,$21 ; 3,32 ; 3,43$ e 3,25 para os tratamentos B, BA, $\mathrm{M}$ e BS, respectivamente (Tabela 1 ). A CC do grupo $\mathrm{M}$ foi significativamente superior aos demais tratamentos $(\mathrm{P}<0,01)$. A CC entre os grupos B e BS não diferiu $(\mathrm{P}>0,05)$, sendo a $\mathrm{CC}$ do lote $\mathrm{BA}$ intermediária entre esse último tratamento e o tratamento $\mathrm{M}$. A redução da CC em comparação à avaliação da CCJ ao início dos tratamentos é resultado da redução do GMD antes do início dos tratamentos. Os GMD obtidos na brachiária estão abaixo de $0,350 \mathrm{~kg}$, o qual permitiria obter pesos ao acasalamento entre 290-310 kg aos 18 meses de idade.

FOX et al. (1988), ao trabalharem com raças com peso adulto de $467 \mathrm{~kg}$ e peso ótimo ao primeiro cio de $297 \mathrm{~kg}$, registraram GMD de 0,466 kg/dia para atingir o primeiro cio aos 14,5 meses de idade. O ganho médio de $0,229 \mathrm{~kg} /$ dia realizado neste trabalho no período seco foi somente $65 \%$ do GMD alvo (0,350 kg/dia). MOSS (1993), na Austrália, destaca que os GMD de novilhas, inferiores a $0,250 \mathrm{~kg}$ no período seco, são devidos ao alto teor de fibra, à baixa digestibilidade e baixa relação proteína/energia em forragens tropicais. Este autor cita que suplementações podem elevar o GMD neste período. Os ganhos dos tratamentos M e BA diferiram estatisticamente dos tratamentos B e BS $(\mathrm{P}<0,01)$, em 
concordância com os resultados de EUCLIDES e EUCLIDES FILHO (1997) e THIAGO et al. (1997).

Os pesos vivos médios alcançados em 14/10/96 foram de 208,3;214,7; 218,3 e 205,3 $\mathrm{kg}$ para os grupos B, BA, M e BS, respectivamente. Os tratamentos M e BA foram superiores aos tratamentos BS e B $(\mathrm{P}<0,01)$. Os pesos vivos aos 365 dias de idade das novilhas foram superiores aos pesos de 193,7 $\pm 1,9 \mathrm{~kg}$ obtidos por SILVA et al. (1987), dados que se referem ao desempenho ponderal da raça Nelore no Estado de São Paulo, coletados entre 1976 a 1981 em 30 fazendas com animais criados exclusivamente a pasto. MARIANTE e ZANCANER (1985) registraram pesos vivos de $199 \pm 1,7 \mathrm{~kg}$ na Fazenda Bonsucesso com dados de 1962 e 1976 de um rebanho Nelore em piquetes de pastos de Panicum maximum cv. Colonião. Já CORRÊA (1994) na Fazenda Modelo CNPGCEMBRAPA com dados de nove anos (1983-1991), em pastos nativos de gramíneas dos gêneros Paspalum, Axonopus, Andropogon, Rinchelytrum e Sporobulus e pastos cultivados de gramíneas de Brachiaria decumbens, B. brizantha cv. Marandu e B. humidicola, e ELER et al. (1989), em 9281 fêmeas da raça Nelore criados em regime exclusivo de pasto em 37 rebanhos do Estado de São Paulo com dados de animais nascidos de 1976 e 1984, verificaram pesos entre, respectivamente, $167,4 \pm 18,2$ e $189 \mathrm{~kg}$.

As CC nos quatro sistemas de alimentação em outubro/96 foram, respectivamente, de 3,51; 3,59; 3,64, e 3,46 para os grupos B, BA, M e BS. A CC dos tratamentos $\mathrm{Me} \mathrm{BA}$ foi significativamente superior à dos tratamentos $\mathrm{B}$ e $\mathrm{BS}(\mathrm{P}<0,01)$, os quais não diferiram entre si $(\mathrm{P}>0,05)$. As diferenças entre as CC são o resultado dos GMD superiores dos tratamentos $\mathrm{M}$ e BA no período em relação aos dos tratamentos B e BS. Na Tabela 2 são apresentados o ganho diário durante o período das águas de 14/10/96 a 03/03/97, o peso vivo (PV) e a condição corporal no início do acasalamento. Os GMD não diferiram estatisticamente entre si $(P>0,05)$. Dados de avaliação de ganhos de peso em cultivares e acessos selecionados de Panicum maximum mostraram GMD entre 0,422 e $0,520 \mathrm{~kg}$, conforme JANK (1994), e de $0,580 \mathrm{~kg}$ no ponto máximo do GMD, com disponibilidade de $900 \mathrm{~kg} / \mathrm{ha} / \mathrm{MS}$ verde (EUCLIDES e EUCLIDES FILHO, 1997).

Os grupos B, BA e M não diferiram estatisticamente $(P>0,05)$ entre si, mas foram todos superiores ao grupo $\mathrm{BS}(\mathrm{P}<0,01)$ em relação ao $\mathrm{PV}$. Estes pesos médios são inferiores em aproximadamente $30 \mathrm{~kg}$ do peso à puberdade considerado pelo NRC (1996), o
Tabela 2 - Ganho médio diário (GMD), peso vivo médio (PV) e condição corporal média (CC) durante o período das águas até o início do acasalamento (14/10/ 96-03/03/97)

Table 2 - Live weight daily gain (LDG), average live weight ( $L W)$ and body condition score (BCS) up to the beginning of the mating season (10/14/96-03/03/97)

\begin{tabular}{|c|c|c|c|c|c|}
\hline \multirow[t]{3}{*}{$\begin{array}{l}\text { Parâmetros } \\
\text { Parameters }\end{array}$} & \multicolumn{5}{|c|}{$\begin{array}{l}\text { Tratamentos } \\
\text { Treatments }\end{array}$} \\
\hline & B & BA & M & BS & Médi \\
\hline & $B$ & $B A$ & $M$ & $B S$ & Mean \\
\hline GMD $(\mathrm{kg})$ & $0,427^{\mathrm{A}}$ & $0,397^{\mathrm{A}}$ & $0,351^{\mathrm{A}}$ & $0,355^{\mathrm{A}}$ & A 0,382 \\
\hline $\begin{array}{l}L D G(\mathrm{~kg} / \text { day }) \\
\mathrm{PV}(\mathrm{kg}) \text { 03/03/97 }\end{array}$ & $265,1^{\mathrm{a}}$ & $266,6^{a}$ & $264,0^{\mathrm{a}}$ & $254,4^{\mathrm{b}}$ & 262,5 \\
\hline $\begin{array}{l}\text { Live weight }(\mathrm{kg}) \\
\mathrm{CC}(1-5) 03 / 03 / 97\end{array}$ & $3,85^{\mathrm{b}}$ & $3,91^{\mathrm{a}}$ & $3,79^{\mathrm{b}}$ & $3,76^{\mathrm{b}}$ & 3,71 \\
\hline
\end{tabular}

Body condition score

A Valores seguidos pela mesma letra, na mesma linha, não diferem estatisticamente $(P>0,05)$.

a, b Valores seguidos por letras distintas, na mesma linha, diferem estatisticamente $(P<0,01)$.

A Values followed by the same letter, in the same line, do not statistically differ $(P>$.05).

$a, b$ Values followed by different letters, in the same line, statistically differ $(P<.01)$

qual recomenda $65 \%$ do peso adulto e 20 a $30 \%$ inferiores as recomendações de peso mínimo ao início do acasalamento de fêmeas zebuínas 320 e $300 \mathrm{~kg}$, respectivamente (FONSECA, 1984; FERREIRA et al., 1995). Comparando com os resultados de ALENCAR et al. (1987), que avaliaram a puberdade e o desenvolvimento de bezerras Nelore e Canchim, recriadas em regime exclusivo de pasto no Estado de São Paulo, a diferença em relação ao peso de entrada em reprodução foi $23 \%$ inferior (262 vs. $323 \pm 6$ quilos). Estes mesmos autores relatam que a idade ao primeiro cio e de entrada em reprodução na

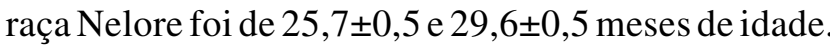

A CC do tratamento BA foi estatisticamente superior $(\mathrm{P}<0,01)$ às dos outros tratamentos. A amplitude dos valores citados por MOSSMAN (1984) para CC em novilhas foram inferiores aos valores de CC observados neste trabalho. FERREIRA et al. (1995) citam uma relação linear entre a maior eficiência reprodutiva em novilhas Nelore e os maiores valores de CC no acasalamento. No entanto, a maioria dos trabalhos somente relaciona a CC ao parto e início do acasalamento com o período de anestro pós-parto de vacas e intervalo parto-concepção.

As idades médias ao início do acasalamento (IAC) não diferiram entre tratamentos $(\mathrm{P}>0,05)$ e foram, respectivamente, 498, 500, 499 e 500 dias para os tratamentos B, BA, M e BS. São raras as publicações com idades médias ao acasalamento de raças zebuínas 
ao redor de 17 meses em sistemas de produção com base em pastagens e sob sistemas extensivos de produção. A necessária redução da idade do acasalamento dos 36-38 meses em fêmeas zebuínas para menores idades, como 17/18 meses, é importante e foi salientada por LOBATO (1995), HILL (1995; 1996), FARIA (1996) e SANCEVERO e FARIA (1998).

Constam da Tabela 3 o ganho diário médio durante o período reprodutivo (GMD), peso (PV) e condição corporal no final do acasalamento (CC) (03/03/97 a 07/05/97). Os GMD não diferiram entre si ( $\mathrm{P}>0,05)$. Os GMD obtidos durante o acasalamento foram $0,109 \mathrm{~kg}$ superiores aos do período anterior (GMD da Tabela 2). O GMD foi superior aos $0,400 \mathrm{~kg}$ descritos por EUCLIDES e VIEIRA (1989) na avaliação de acessos selecionados de Panicum maximum.

O PV do tratamento BS foi inferior $(\mathrm{p}<0,01)$ aos outros três tratamentos estudados. Os PV das novilhas avaliadas foram superiores aos determinados aos 550 dias por MARIANTE e ZANCANER (1985), SILVA et al. (1987) e CORRÊA (1994), que obtiveram, respectivamente, pesos de 259,6 2 2,1 kg em 907 novilhas, $242 \pm 3,1 \mathrm{~kg}$ em 1106 novilhas e $231,8 \pm 5,2 \mathrm{~kg}$ em 406 novilhas. A média de peso vivo de 2531 novilhas Nelore com idade média de 516 dias da Agropecuária Jacarezinho Ltda, nascidas em 1995, foi $255,4 \mathrm{~kg}$ (GENSYS, 1998). A utilização de sistemas forrageiros com maior potencial de ganho para

Tabela 3 - Ganho médio diário (GMD) durante a estação de acasalamento, peso vivo (PV) e condição corporal (CC) ao final da estação de acasalamento (03/03/97 a 07/05/97)

Table 3 - Live weight daily gain (LDG) during mating season, average live weight (LW) and body condition score (BCS) at the end of mating season (03/03/97 to 05/07/97)

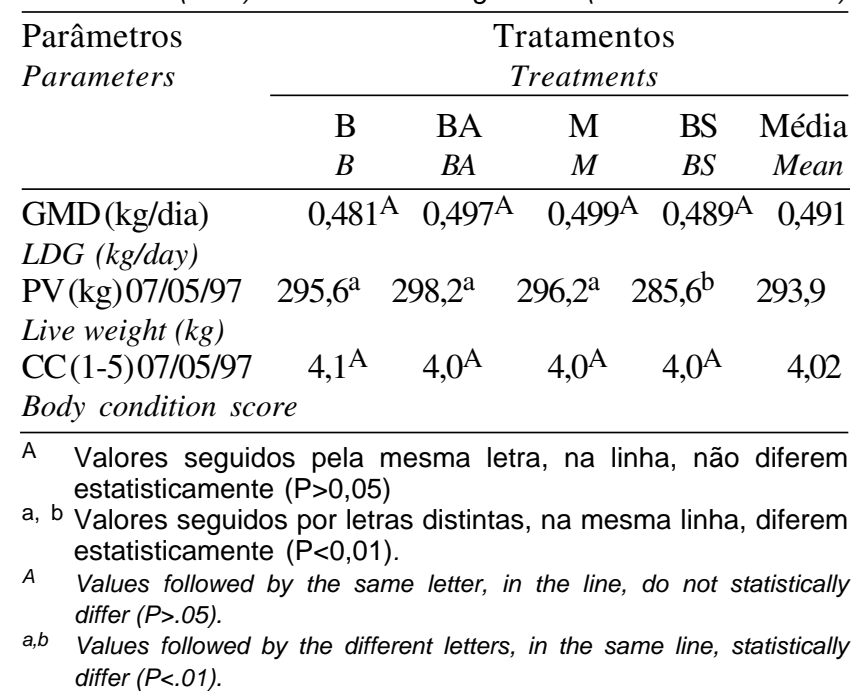

peso, como o Panicum maximum cv Mombaça e/ou a combinação de sistemas de recria que permitam ganhos de 0,200 a $0,300 \mathrm{~kg} /$ dia durante o período seco, foi a responsável pelas diferenças de peso entre as novilhas do experimento e a totalidade de novilhas da raça Nelore nascidas no mesmo estabelecimento na safra de 1995.

Não foi determinada a relação da idade da mãe e do sistema de alimentação no peso pós-desmama (B, BA, M e BS) com a taxa de prenhez obtida pelas novilhas. Os tratamentos B, BA, M e BS utilizados durante a estação seca não afetaram a taxa de prenhez, 22,2;24,2; 20,7 e 13,8\%, respectivamente. A taxa de prenhez média de $20,6 \%$ foi superior aos $4,8 \%$ relatados por FARIA (1996) e SANCEVERO e FARIA (1998). HILL (1995; 1996) cita taxas de prenhez de 30 e $23 \%$. SILVA (1997) obteve $12 \%$ de prenhez em novilhas Nelore aos 14 meses de idade. São escassas as citações de taxas de prenhez aos 18 meses em fêmeas zebuínas em sistemas extensivos, necessitando-se mais pesquisa nesta área. Normalmente as referências nacionais relatam pesos ao início de estação de monta que se referem a fêmeas de 25 a 28 meses de idade no início do período das águas.

A redução da idade do primeiro acasalamento para 24-26 meses de idade com raças britânicas, cruzas zebuínas e zebuínos puros é possível, quando é dado acesso a pastagens de inverno ou quando se fornecem suplementos alimentares nos dois primeiros invernos pós-desmama (BERETTA e LOBATO, 1993; PEREIRA NETO e LOBATO, 1998). Para os objetivos de redução da idade de primeiro serviço, diminuição de custos, elevação da taxa de desfrute e maior eficiência das áreas destinadas à recria de fêmeas na raça Nelore, o acasalamento aos 18 meses de idade é um desafio que tem como meio a obtenção de novilhas mais precoces (HILL, 1995; LOBATO, 1995; ALBUQUERQUE e FRIES, 1997; FRIES e ALBUQUERQUE, 1999). A idade média ao primeiro parto das novilhas prenhas neste estudo foi de 29 meses, representando antecipação de 15,7, 11,8, 14,9 e 6,7 meses em comparação com as idades citadas por MATTOS e ROSA (1984), MARIANTE e ZANCANER (1985), GALINA e ARTHUR (1989) e ALENCAR et al. (1999). A seleção da população e o programa de melhoramento genético aplicado com ênfase na seleção de animais mais precoces na raça Nelore começam a gerar resultados importantes para as produções pecuárias de ciclo curto nas regiões subtropicais e tropicais. Como os pesquisadores citados, FRIES e ALBUQUERQUE (1999) indicam que a decisão/oportunidade de utilização de fêmeas sexual- 
Rev. bras. zootec.

mente mais precoces terá reflexo direto na eficiência, rentabilidade, taxa de desfrute e competividade da pecuária de corte nacional.

$\mathrm{Na}$ Tabela 4 é apresentado o resumo do desempenho geral das variáveis de peso vivo PN, PJ, PA, PO, PIA, PFA, CCJ, CCA, CCO, CCI, CCR, IAC, GDI, GDS $_{12}$, nascimento-acasalamento, GDM, Cs, Ps e Ms entre novilhas Nelore diagnosticadas prenhas e falhadas.

Foram determinadas $(\mathrm{P}<0,01)$ diferenças significativas entre os pesos vivos das novilhas que viriam a prenhar ou a falhar no primeiro acasalamento medidos no início dos tratamentos (PJ), em agosto (PA) e em outubro (PO), início (PIA) e final do acasalamento (PFR). As diferenças médias foram, respectivamente, de $10,12,13,516$ e $16,6 \mathrm{~kg}$. Conclui-se que novilhas de maior peso vivo nos períodos citados e maior ganho de peso no período de suplementação e do nascimento ao início da monta alcançaram prenhez ao sobreano. No entanto, o peso vivo ao sobreano não deve ser o único parâmetro determinante da idade à puberdade, da maturidade sexual e concepção de novilhas. GREER et al. (1983) e PATTERSON et al. (1992) reforçam não ser a puberdade determinada por um peso per si, mas sim por uma série de eventos fisiológicos que ocorrem com determinado peso. Neste experimento, diferença média de $13,7 \mathrm{~kg}$ a favor das novilhas prenhes para PJ, PO, PIA e PFR produziu taxa de prenhez média de 20,6\%. A amplitude de peso vivo no PIA entre as novilhas Nelore prenhes foi de 227 a $336 \mathrm{~kg}$, sendo que $70 \%$ dessas novilhas prenhes possuíam entre 265 e $336 \mathrm{~kg}$. Pode-se concluir que o descarte das novilhas muito leves à desmama e ao sobreano pode ser medidas de manejo e seleção para a elevação dos índices de prenhez aos 18 meses de idade de novilhas Nelore.

A CC das novilhas prenhas foi, em média, 0,11 superior à das novilhas falhadas (Tabela 4). As diferenças em CC oscilaram entre 0,09 e 0,12 $(\mathrm{P}<0,01)$. Novilhas que ficaram prenhas ou falhadas diferiram $(\mathrm{P}<0,01)$ em $\mathrm{CC}$ em diferentes momentos do trabalho. VESERAT et al. (1996) determinaram maior prenhez nos primeiros 21 dias de acasalamento em novilhas de maior CC e peso vivo. Neste experimento, as novilhas prenhas foram 14 dias mais velhas $(\mathrm{P}<0,01)$ que as falhadas ao início do acasalamento, o que está de acordo com as observações de BERGMANN e HOHENBOKEN (1992). O peso vivo e a idade ao início da estação do acasalamento são importantes na prenhez de novilhas, principal-
841

Tabela 4 - Desempenho de novilhas prenhas e falhadas aos 17/18 meses de idade durante o período experimental em relação ao peso vivo, ganho médio diário, idade ao início do acasalamento, condição corporal e escores visuais de conformação, precocidade e musculosidade ao sobreano

Table 4 - Summary of the performance of pregnant and open heifers bred at 17/18 months of age regarding liveweight, liveweight daily gain, age at the beginnig of the mating season, body condition score and yearling visual scores concerning conformation, precocity and muscularity

\begin{tabular}{|c|c|c|}
\hline $\begin{array}{l}\text { Parâmetros } \\
\text { Parameters }\end{array}$ & $\begin{array}{c}\text { Prenha } \\
\text { Pregnant }\end{array}$ & $\begin{array}{c}\text { Falhada } \\
\text { Open }\end{array}$ \\
\hline \multicolumn{3}{|l|}{ Peso vivo (kg) } \\
\hline \multicolumn{3}{|l|}{ Live weight (kg) } \\
\hline Nascimento (PN) & 28,77 & $29,28^{*}$ \\
\hline \multicolumn{3}{|l|}{ Birthweight } \\
\hline Inicial 31/05/96(PJ) & 187,42 & $177,42^{* *}$ \\
\hline \multicolumn{3}{|l|}{ Initial } \\
\hline Inicial 06/08/96(PA) & 202,11 & $189,84^{* *}$ \\
\hline \multicolumn{3}{|l|}{ Initial } \\
\hline Inicial 14/10/96(PO) & 221,64 & $208,14^{* *}$ \\
\hline \multicolumn{3}{|l|}{ Initial } \\
\hline \multicolumn{3}{|l|}{ 03/03/97(PIA) } \\
\hline \multicolumn{3}{|l|}{ Starting mating } \\
\hline \multicolumn{2}{|l|}{ 07/05/97(PFR) } & $289,91^{* *}$ \\
\hline \multicolumn{3}{|l|}{ End of mating } \\
\hline \multicolumn{3}{|l|}{ Condiçãocorporal(1-5) } \\
\hline \multicolumn{3}{|l|}{ Body condition score } \\
\hline 31/05/96(CCJ) & 3,94 & $3,84^{* *}$ \\
\hline 06/08/96(CCA) & 3,41 & $3,29^{* *}$ \\
\hline 14/10/96(CCO) & 3,64 & $3,54^{* *}$ \\
\hline 03/03/97(CCI) & 3,90 & $3,81^{* *}$ \\
\hline 07/05/97(CCR) & 4,12 & $4,01^{* *}$ \\
\hline \multicolumn{3}{|l|}{ Idade acasalamento (dias)(IAC) } \\
\hline Age at mating (days) & 511 & $497^{* *}$ \\
\hline \multicolumn{3}{|l|}{ Ganho médio diário $(\mathrm{kg})$} \\
\hline \multicolumn{3}{|l|}{ Live weight daily gain $(\mathrm{kg})$} \\
\hline Nascimentoaté31/05/96 (GMD) & 0,665 & $0,661^{\mathrm{NS}}$ \\
\hline \multicolumn{3}{|l|}{ Birth until 05/31/96 } \\
\hline 31/05/96até14/10/96(GMD) & 0,245 & $0,225^{* *}$ \\
\hline Nascimento até início acasalamento & 0,483 & $0,461^{\text {** }}$ \\
\hline \multicolumn{3}{|l|}{ Birth to beginning of mating } \\
\hline Acasalamento(GMD) & 0,487 & $0,490^{\mathrm{NS}}$ \\
\hline \multicolumn{3}{|l|}{ Mating season } \\
\hline \multicolumn{3}{|l|}{ Escore visual ao sobreano (1-5) } \\
\hline \multicolumn{3}{|l|}{ Yearling visual scores } \\
\hline Conformação (Cs) & 3,42 & $2,96^{* *}$ \\
\hline Conformation & & \\
\hline Precocidade (Ps) & 3,58 & $3,16^{* *}$ \\
\hline Precocity & & \\
\hline $\begin{array}{l}\text { Musculosidade (Ms) } \\
\text { Muscularity }\end{array}$ & 3,17 & $2,79^{* *}$ \\
\hline
\end{tabular}

NS Diferençanão-significativa $(P>0,05)$ (Nonsignificantdifference $[P>$.05]).

* Diferença significativa $(P<0,05)$ (Significant difference $[P<.05]$ ).

** Diferença significativa $(P<0,01)$ (Significant difference $[P<.01]$ ). 
mente com parições aos dois anos e meio.

Os valores médios dos escores de conformação (Cs), precocidade (Ps) e musculosidade (Ms) ao sobreano das novilhas que alcançaram prenhez também foram superiores aos das novilhas falhadas $(\mathrm{P}<0,01)$. A ponderação dos escores visuais de conformação, precocidade e musculosidade na composição de índices de seleção com as características de ganho de peso tem permitido a identificação de características associadas com maior precocidade, como a capacidade de deposição de gordura, massas musculares mais desenvolvidas e tamanho moderado. COSTA PITA et al. (1998) também encontraram maior frequência de escores visuais 3 a 5 de conformação, precocidade e musculosidade em novilhas Nelore prenhes aos 18 meses em relação as prenhes aos 24-26 meses de idade e as falhadas aos 24-26 meses, respectivamente, 93,1; 99,1 e 96,6\% (precoce) versus 85,7, 92,4 e $82 \%$ (normal) e $72,4,83$ e $67,8 \%$ (falhada). Os resultados obtidos mostram que os escores visuais ao sobreano podem auxiliar na indicação da idade ao primeiro parto de fêmeas Nelore.

\section{Conclusões}

Os sistemas alimentares Brachiária + Anipro® ${ }^{\circledR}$ e Milheto permitiram maior ganho médio diário, maior peso vivo e melhor condição corporal ao final do período de suplementação.

Os sistemas alimentares de recria (Brachiária, Brachiária + Anipro® ${ }^{\circledR}$ e Milheto) determinaram maiores valores no parâmetro peso vivo em relação ao sistema Brachiária + Sal Ouro ${ }^{\circledR}$ no período das águas.

Os sistemas alimentares de recria no período de acasalamento não determinaram diferenças em ganho médio diário e condição corporal. No mesmo período, o peso vivo foi superior para os sistemas Brachiária, Brachiária + Anipro $\AA^{B}$ e Milheto em relação a Brachiária + Sal Ouro ${ }^{\circledR}$.

Os sistemas alimentares de recria no período de seca e a idade da mãe da novilha não afetaram o desempenho reprodutivo de novilhas postas em serviço, aos 17/18 meses de idade.

As novilhas prenhes no acasalamento aos $17 / 18$ meses de idade foram mais pesadas, mais velhas e tiveram melhor condição corporal, maiores ganhos médios diários nos períodos de seca e águas e maiores escores visuais de conformação, precocidade e musculosidade ao sobreano do que as falhadas.

\section{Agradecimento}

À Agropecuária Jacarezinho Ltda e ao GenSys Consultores Associados S/C Ltda, pelo apoio e auxílio na realização deste trabalho de pesquisa.

\section{Referências Bibliográficas}

ALBUQUERQUE, L.G., FRIES, L.A. Precocidade: estratégia de seleção. In: SIMPÓSIO: O NELORE DO SÉCULO XXI NELORE PRECOCE: SELEÇÃO, PRODUÇÃO E COMERCIALIZAÇÃO, 4., 1997, Uberaba. Anais... Uberaba: ABCZ-ACNB, 1997. p.164-179.

ALENCAR, M.M., OLIVEIRA, J.A.L., ALMEIDA, M.A. 1999. Idade ao primeiro parto, peso ao parto e desempenho produtivo de vacas Nelore e cruzadas Charolês x nelore. Rev. bras. zootec., 28(4):681:686.

ALENCAR, M.M., COSTA, J.L., CORRÊA, L.A. 1987. Desempenho produtivo de fêmeas das raças Canchim e Nelore. I. Desenvolvomento e Puberdade. Pesq. Agropec. Bras., 22(7):753-758.

BERETTA, V., LOBATO, J.F.P. Efeito do nível alimentar até o primeiro acasalamento na eficiência reprodutiva e produtiva de novilhas de corte. In: REUNIÃO ANUAL DA SOCIEDADE BRASILEIRA DE ZOOTECNIA, 30, 1993, Rio de Janeiro. Anais... Rio de Janeiro: SBZ, 1993. p.181.

BERGMANN, J.A.G., HOHENBOKEN, W.D. 1992. Prediction of fertility from calfhood traits of Angus and Simenthal heifers. J. Anim. Sci., 70(8):2611-2621.

CORRÊA, E.S. Avaliação dos desempenhos reprodutivo e produtivo em um sistema de produção de gado de corte: Jaboticabal, SP: UNESP, 1994, 106p. Dissertação (Mestrado em Zootecnia) - Universidade Estadual Paulista, 1994.

COSTA PITA, F.V., FRIES, L.A., ALBUQUERQUE, L.G. Utilização de escores visuais como indicadores da idade à maturidade sexual de novilhas Nelore. In: SIMPÓSIO NACIONAL DE MELHORAMENTO ANIMAL, 2, 1998, Viçosa. Anais... Viçosa: SBMA, 1998. p.383-384.

DUARTE, C.M.L. Avaliação de forrageiras perenes de verão e milheto (Pennisetum americanum (L.) Leeke) cv. comum integrados em sistemas de produção animal em pastagens: Porto Alegre, RS: UFRGS, 1980. 150p. Tese (Mestrado em Agronomia-Forragicultura) - Universidade Federal do Rio Grande do Sul, 1980.

ELER, J.P., LOBO, R.B., ROSA, A.N. 1989. Influências de fatores genéticos e de meio em pesos de bovinos da raça Nelore criados no estado de São Paulo. R. Bras. Zootec., 18(2):103-111.

EUCLIDES, V.P.B., EUCLIDES FILHO, K. Avaliação de forrageiras sob pastejo. In: SIMPÓSIO SOBRE AVALIAÇÃO DE PASTAGENS COM ANIMAIS, 1., 1997, Maringá. Anais... Maringá: CCA/UEM, 1997. p.85-111.

EUCLIDES, V.P.B., VIEIRA, A. Avaliação de cultivares de Panicum maximum sob pastejo. In: REUNIÃO ANUAL DA SOCIEDADE BRASILEIRA DE ZOOTECNIA, 26, 1989, Porto Alegre. Anais... Porto Alegre: SBZ, 1989. p.97.

FARIA, N.R. Grandes projetos agropecuários 1996. Carne, sistema de produção, custos, comercialização. R. Bras. Reprod. Anim., 20(3):106-111.

FERREIRA, M.B.D., SATURNINO, H.M., SILVA FILHO, J.M. et al. Efeitos de peso e condição corporal na eficiência reprodutiva de novilhas zebu. In: CONGRESSO BRASILEIRO DE REPRODUÇÃO ANIMAL, 11, 1995, Belo Horizonte. Anais... Belo Horizonte: CBRA, 1995. p.376. 
Rev. bras. zootec.

FERREL, C.L., JENKINS, T.G. 1988. Influence of biological types on energy requirements. Agricultural Research Service and United States Department of Agriculture. p.86-90. (Beef research progress report, 3).

FONSECA, V.O. 1984. O manejo da reprodução e o aumento da eficiência reprodutiva do zebu. Inf. Agropec., 10(112):56-68.

FOX, D.G., SNIFFEN, C.J., O'CONNOR, J.D. 1988. Adjusting nutrient requirements of beef cattle for animal and enviromental variations. J. Anim. Sci., 66(6):475-495.

FRIES, L.A. 1998. Genética de gado de corte orientada para lucratividade. In: LOBATO, J.F.P., BARCELLOS, J.O.J., KESSLER, A.M. (Eds.) Produção de bovinos de corte. Porto Alegre: EDI-PUCRS, 1.ed. p.193-234.

FRIES, L.A., ALBUQUERQUE, L.G. Prenhez aos catorze meses: Presente e Futuro. Elementos do componente genético. In: REUNIÃO ANUAL DA SOCIEDADE BRASILEIRA DE ZOOTECNIA, 36, 1999, Porto Alegre. Anais... Porto Alegre: SBZ, 1999. p.227-249.

GALINA, C.S., ARTHUR, G.H. 1989. Review of cattle reproduction in the tropics. Part 1 . Puberty and age at first calving. Anim. Breed. Abstr., 57(7):583-590.

GARDNER, A.L. 1986. Técnicas de pesquisa em pastagem e aplicabilidade de resultados em sistemas de produção. Brasília: IICA/EMBRAPA-CNPGL. 197p (Série publicações miscelâneas, 634).

GENSYS CONSULTORES ASSOCIADOS Ltda S/C -1998. Registros Gensys. Porto Alegre: Gensys. 2p.

GOMES, F.P. 1985. Curso de estatística experimental. Piracicaba: Editora Nobel. 466p.

GREER, R.C., WHITMAN, R.W., STAIGMILLER, R.B. et al. 1983. Estimating the impact of management decisions on the ocurrence of puberty in beef heifers. J. Anim. Sci., 56(1):30-39.

HILL, I.D. A resposta do gado Nelore à seleção. In: SYMPOSIUM: O NELORE DO SÉCULO XXI, 3, 1995, Ribeirão Preto. Anais... Ribeirão Preto: ACNB, 1995. p.49-61.

HILL, I.D. 1996. Precocidade: O caminho do lucro. R. Bras. Reprod. Anim., 20(3):99-105.

JANK, L. Potencial do Gênero Panicum. In: SIMPÓSIO BRASILEIRO DE FORRAGEIRAS E PASTAGENS, Campinas, Anais... Campinas: CNBA, 1994. p. 25-32.

LEITE, G.G., EUCLIDES, V.P. Utilização de pastagens de Brachiaria spp. In: SIMPÓSIO SOBRE MANEJO DE PASTAGEM, 11, 1994, Piracicaba. Anais... Piracicaba: ESALQ/FEALQ, 1994. p.267-298.

LOBATO, J.F.P. Produção e manejo de gado de corte. In: REUNIÃO ANUAL DA SOCIEDADE BRASILEIRA DE ZOOTECNIA, 32, 1995, Brasília. Anais... Brasília: SBZ, 1995. p.405-414.

LOBATO, J.F.P. 1998. Considerações efetivas sobre seleção, produção e manejo para maior produtividade de rebanhos de cria. In: LOBATO, J.F.P., BARCELLOS, J.O.J., KESSLER, A.M. (Eds.) Produção de bovinos de corte. Porto Alegre: EDI-PUCRS, 1.ed. p.405-414.

LOWMAN, B.G. SCOTT, N., SOMERVILLE, S. 1976. Condition scoring beef cattle. Edinburgh: East of Scotland College of Agriculture. 8p. (Bulletin 6).

MARASCHIN, G.E. 1981. Oportunidade para uma eficiente produção animal baseada em pastagens. Lav. Arrozeira., p.10-20.

MARIANTE, A da S., ZANCANER, A. 1985. Crescimento $e$ reprodução em gado Nelore - Visão do criador e de pesquisador. São Paulo: Editora dos Criadores. 158p.

MATTOS, S., ROSA, A.N. 1984. Desempenho reprodutivo de fêmeas de raças zebuínas. Infor. Agropec., 10(112):29-34.

MORAES, A. Pressões de pastejo e produção animal em milheto (Pennisetumamericanum (L.) Leeke): Porto Alegre, RS: UFRGS, 1984. 104p. Dissertação (Mestrado em Agronomia) - Universidade Federal do Rio Grande do Sul, 1984.
MORAES, A., MARASCHIN, G.E., NABINGER, C. Pastagens nos ecossistemas de clima subtropical Pesquisas para o desenvolvimento sustentável. In: REUNIÃO ANUAL DA SOCIEDADE BRASILEIRA DE ZOOTECNIA. SIMPÓSIO PASTAGENS NOS ECOSSISTEMAS BRASILEIROS PESQUISA PARA O DESENVOLVIMENTO SUSTENTÁVEL, 32, 1995, Brasília. Anais... Brasília: SBZ, 1995. p.147-200.

MOSS, R.S. 1993. Rearing heifers in the subtropics and tropics: nutrient requirements and supplementation. Trop. Grassl., 27(3):238-249.

MOSSMAN, D.H. Management of beef cows for optimum reproduction. In: BEEF CATTLE REPRODUCTION-ANIMAL INDUSTRIES WORKSHOP, LINCOLN COLLEGE WORKSHOP, 1984, Cantenbury. Proceedings... Cantenbury: Lincoln College, 1984. p.71-82.

NATIONAL RESEARCH COUNCIL - NRC. 1984. Nutrient requirements of beefcattle. 6 ed. Washington D.C.: National Academy Press. p.90.

NATIONAL RESEARCH COUNCIL - NRC. 1996. Nutrient requirements of beef cattle. 7.ed. Washington D.C.: National Academy Press. p.242.

PATTERSON, D.J., PERRY, R.C. KIRACOFE, G.H. et al. 1992. Management considerations in heifer development and puberty. J. Anim. Sci., 70(12):4018-4035.

PEREIRA, J.C.C. 2000. Contribuição genética do zebu na pecuária bovina do Brasil. Infor. Agropec., 21(205):30-38.

PEREIRA NETO, O.A., LOBATO, J.F.P. 1998. Efeitos da ordem de utilização de pastagens nativas melhoradas no desenvolvimento e comportamento reprodutivo de novilhas de corte. R. Bras. Zootec., 27(1):60-65.

SANCEVERO, A.B., FARIA, N.R. Produção do novilho precoce e super precoce. In: SIMPÓSIO NACIONAL DE MELHORAMENTO ANIMAL, 2, Uberaba, 1998. Anais... Uberaba: SBMA, 1998. p.185-196.

SEVERO, J.L.P. 1994. Manejo e controle de produção para a implantação de um programa de melhoramento genético de bovinos de corte. In: Bovinos de corte: seleção e cruzamento. Porto Alegre: GenSys Consultores Associados S/C Ltda. 80p.

SILVA, L.O.C., ROSA, A.N., NOBRE, P.R.C. et al. 1987. Análise de pesos de bovinos Nelore criados à pasto no estado de São Paulo, Brasil. Pesq. Bras. Agropec., 22(11/12):1245-1256.

SILVA, P.R.S. 1997. Tirando vantagens da precocidade sexual. In: ANUALPEC 97. Anuário da pecuária brasileira. São Paulo: Argus Comunicação. p.34-39.

STATISTICAL ANALISYS SYSTEM - SAS. 1988. Language guide for personal computer, 6.08, Cary: SAS Institute, v. 12, 378p.

VERESAT, G.M., OLTEN, J.M., TASSEL, W. Evaluating puberty in beef heifers raised for fall calving in a Mediterranean environment in the northern hemisphere. In: INTERNATIONAL CONGRESS ON ANIMAL REPRODUCTION, 13, 1996, Sydney. Proceedings... Sydney: CSIRO, v.3, 1996. p.13-19.

ZIMMER, A.H. Produção de carne em pastos de capins do Gênero Brachiaria. In: ENCONTRO SOBRE CAPINS DO GENERO BRACHIÁRIA, 1, 1987, Nova Odessa. Anais... Nova Odessa: Instituto de Zootecnia, 1987. p.253-294.

Recebido em: 04/04/00 Aceito em: 24/01/01 